ORIGINAL ARTICLE

\title{
Dynamic force distribution during level walking under the feet of patients with chronic ankle instability
}

\author{
M Nyska, S Shabat, A Simkin, M Neeb, Y Matan, G Mann
}

See end of article for authors' affiliations .....................

Correspondence to: Dr Shabat, Department of Orthopaedic Surgery, Foot and Ankle Service, Sapir Medical Center, 48

Tchernichovsky' St, KfarSaba 44281, Israel; drshabat@hotmail.com

Accepted

16 December 2002

\begin{abstract}
Objectives: To examine changes in the pattern of force transfer between the foot and the floor associated with chronically sprained ankles by measuring the peak forces and their timing under several regions of the feet during level walking.

Methods: Twelve young male subjects (mean (SD) age 21 (2) years) with recurrent ankle sprains were studied. Seven of them had unilateral and bilateral chronic instability and laxity, and five had bilateral instability. Twelve healthy men (without orthopaedic or medical disease) served as a control group. Subjects walked at their own pace along a $7 \mathrm{~m}$ walkway, which included a Mini-EMED pressure distribution measuring system. The variables measured were relative peak force (fraction of body weight) and relative timing (fraction of stance time). These variables were measured under six regions of interest in each foot print: heel, midfoot, medial, central, and lateral forefoot, and toes.

Results: (a) A significant delay to the time of peak force under the central and lateral forefoot and toes in subjects with chronic ankle instability. (b) A significant decrease in the relative forces under the heel and toes and an increase in the relative forces under the midfoot and lateral forefoot in subjects with chronic ankle instability. (c) In the patients with unilateral instability, there were no significant differences in any of the variables between the injured and non-injured foot.

Conclusions: In patients with chronic ankle instability, there is a slowing down of weight transfer from heel strike to toe off, a reduced impact at the beginning and end of the stance phase, and a lateral shift of body weight.
\end{abstract}

A nkle sprain is the most common injury in athletes and people participating in sport, representing $15-20 \%$ of all sport injuries. ${ }^{1}$ In soccer and basketball, the risk of ankle sprain is even higher, being $45 \%$ and $31 \%$ respectively. ${ }^{2}$ Half of the general population has at least one ankle sprain during life. In military recruits there is increased risk, mainly during sport. ${ }^{3}$ About $20 \%$ of patients with an acute sprain will have residual complaints depending on the severity of the initial trauma. ${ }^{45}$

There are intrinsic and extrinsic risk factors for chronic instability of the ankle. Among the latter are the type of physical activity, type of ground, and type of shoe wear. ${ }^{6}$ Ankle sprains account for $25-50 \%$ of injuries in sports that include running and jumping, such as basketball, volleyball, soccer, and football. ${ }^{7}$ It is the most common injury sustained by the cadets at the United States Military Academy, West Point: about one third have one or more ankle sprains during the four year programme, and about 350 of these injuries are evaluated and treated in their athletic injury programme each year. More than $50 \%$ of these injuries are moderate to severe and prevent the cadets from resuming full activity for at least two weeks. ${ }^{3}$ Uneven ground may increase the susceptibility of soldiers to acute sprains. Traditionally soldiers wear high top military boots to prevent ankle sprains. However, it has been shown that they do not have any advantage over high basketball shoes. ${ }^{8}$ Intrinsic conditions associated with recurrent ankle sprain may be congenital, such as tarsal coalition ${ }^{9}$ and hyperlaxity of joints. ${ }^{10}$ It was found that military recruits had more ankle sprains if the body mass moment of inertia was high and if they had a history of sprain. ${ }^{8}$

Chronic ankle instability is assessed clinically and radiologically. Radiological evaluation includes mainly stress views of the ankle and may confirm mechanical laxity. ${ }^{11}$ However, there is a low correlation between stress radiographs and functional status, and patients are usually defined as having functional instability based on their subjective complaint of the ankle joint "giving way".1213 The possible causes of functional instability of the ankle include proprioceptive disorder, ${ }^{12}$ muscle weakness, and subtalar instability. ${ }^{14}$ It seems that the leading cause is nerve injury within or proximal to the lateral ligament. This injury may reduce joint and skin sensation, weaken peroneal muscles, interfere with joint proprioception, balance, and posture stability, and elongate nerve conduction and reaction times, causing repeated ankle injury with or without demonstrable ankle instability. ${ }^{15}$ Lofvenberg et al ${ }^{16}$ have shown that delayed proprioceptive response to sudden angular displacement of the ankle may predispose to chronic lateral instability of the ankle. We hypothesise that patients with recurrent ankle sprains may have a typical altered gait pattern, which may be related to the altered connection between the central system and the injured muscle and/or nerves surrounding the ankle. This may play a role in the development of chronic ankle instability and laxity.

\section{MATERIALS AND METHODS}

Twelve male subjects with recurrent ankle sprain (more than three in six months) were studied using the mini-EMED plantar foot pressure system. Twelve normal healthy subjects, without any medical or orthopaedic diseases, served as a control group. We analysed the changes in the pattern of force transfer and peak forces under the feet during level walking in patients with chronic instability of the ankle (CI) compared with the control group.

Evaluation of gait may be performed using three dimensional motion analysis systems, force plates, or various plantar foot pressure distribution systems. The mini-EMED is a commercially available electronic system for recording and evaluating the distribution of pressures on the plantar aspect of the foot. The overall size of the pressure platform is 
$57 \times 32 \mathrm{~cm}$, with a sensing area of $684 \mathrm{~cm}^{2}$, set flush into a walkway $7 \mathrm{~m}$ long. The pressure plate has four sensors $/ \mathrm{cm}^{2}$ with an accuracy of $\pm 5 \%$ and a sampling rate of $50 \mathrm{~Hz}$. The maximal total force that can be applied is $86900 \mathrm{~N}$. All measurements were taken with the subjects walking barefoot over the pressure plate. Three walks for each foot (six walks for each subject) were recorded and printed. Each print consisted of a time peak-force curve for six regions of interest on the foot. The regions of interest, which were analysed automatically by the system software, were the heel, midfoot, lateral, central, and medial forefoot, and the toes. The time variables were calculated as the ratio of time from the start of the stance to peak force under the region of interest and the total stance time. The force variables were calculated as the ratio of the peak force under the region of interest to the body weight.

Five of the patients with CI had bilateral recurrent sprain. Seven had unilateral sprains, five on the left and two on the right. In each subject in the CI group, the two legs were compared in order to record differences in pattern, if any, between the five patients with bilateral sprains and the seven patients with unilateral sprains. The CI and control groups were then compared. Overall, 24 feet were examined in each group, among them 17 injured in the CI group.

\section{Statistical analysis}

Means (SD) were calculated for the different time and force variables. The differences within the groups and between the groups were compared using one way analysis of variance. Differences of $\mathrm{p}<0.05$ were considered significant.

\section{RESULTS}

The mean age of the CI group was 20.8, and that of the control group was 23.4 years. The CI group was heavier by about $5 \mathrm{~kg}$, but height and body mass were similar (table 1 ).

There was a significant delay to the time of peak force under the central forefoot, lateral forefoot, and toes in the CI group (table 2). There was significantly longer contact time of the heel and midfoot areas in the CI group.

There was a significant decrease in relative forces under the heel and toes and increase in relative forces under the midfoot and lateral forefoot in the CI group compared with the controls (table 3 ). There were no significant differences in any of the variables between the injured and the non-injured foot in the patients with unilateral CI.

Tables 2 and 3 give results for the 17 injured feet in the CI group compared with the 24 "normal feet" in the control group. As there was no significant difference between the right and left foot in the group with bilateral injuries and, even more importantly, in the group with unilateral injury (as one could have assumed), the results would not have changed statistically if we had presented results for all 24 feet in the CI group compared with the 24 feet of the control group.

\section{DISCUSSION}

Patients with CI had a different gait pattern from normal subjects. The longer duration of contact of the heel to central

Table 1 Basic data on patients with chronic instability of the ankle and controls

\begin{tabular}{llll}
\hline & Controls & Injured & p Value \\
\hline Number of patients & 12 & 12 & - \\
Age (years) & $23.4(3.8)$ & $20.8(1.5)$ & $<0.01$ \\
Weight $(\mathrm{kg})$ & $72.3(9.2)$ & $77.4(9.0)$ & $\mathrm{NS}$ \\
Height $(\mathrm{cm})$ & $180.4(6.5)$ & $181.0(6.3)$ & $\mathrm{NS}$ \\
\hline
\end{tabular}

Values are mean (SD).
Table 2 Time variables (percentage of total stance time) in patients with chronic instability of the ankle and controls

\begin{tabular}{llll}
\hline & Controls & Injured & p Value \\
\hline Number of patients & 12 & 12 & - \\
Number of feet examined & 24 & 17 & - \\
Central forefoot & $17.88(1.68)$ & $18.83(1.22)$ & $<0.01$ \\
Lateral forefoot & $15.47(1.98)$ & $16.87(2.04)$ & $<0.05$ \\
Toes & $16.56(0.42)$ & $17.03(0.38)$ & $<0.05$
\end{tabular}

Values are mean (SD).

forefoot indicates a slowing down of weight transfer from heel strike to toe off. We speculate that this is because they are hesitating to put weight on the forefoot. The main factor in this longer contact time was found to be the delay to peak force under the central and lateral forefoot and the toes, which are in contact with the ground towards the end of the stance phase. The position of the foot at the end of the stance phase, which is in plantar flexion, is inherently less stable than at the beginning of the stance when the foot is in dorsiflexion. The shape of the talus, which is wide anteriorly and narrows posteriorly, inherently gives less stability while the leg is plantar flexed. It has been shown that examining the drawer sign of the ankle in $15^{\circ}$ of plantar flexion gives a larger anterior displacement. ${ }^{11}$ The slowing down towards the end of the stance phase may indicate compensatory mechanisms, which give the foot enough time to stabilise. The greater forces under the midfoot and lateral forefoot indicate lateral shift of the centre of pressure. This lateral shift while the ankle is in its most unstable position of plantar flexion may increase the susceptibility of the ankle to sprain. Becker et $a l^{17}$ found in a similar study that patients with functional instability also showed significantly increased lateral loading of the unstable foot. They suggested this to be due to reduced peroneal strength during the stance phase as the result of a proprioceptive deficit caused by trauma.

The similarity of the distribution of plantar foot pressure of the two legs of patients with unilateral CI may suggest that the central pattern controlling the stance is different from that in normal subjects, defining people "at risk" of developing CI. Louwerens et al ${ }^{18}$ evaluated the electromyographic activity of the peroneus longus and anterior tibial muscles of 25 patients with CI. No significant difference was found between the symptomatic and asymptomatic leg of patients with unilateral instability under the same walking conditions. These findings suggest changes in the central control.

In conclusion, patients with CI have a different pattern of walking. They hesitate towards the end of the stance phase and tend to put a greater load on the lateral forefoot, causing a lateral shift of the centre of pressure. There is no difference between the injured and non-injured leg, suggesting that the central control of walking is altered. This requires further examination.

Table 3 Force variables in patients with chronic instability of the ankle and controls

\begin{tabular}{llll}
\hline & Controls & Injured & p Value \\
\hline Number of patients & 12 & 12 & - \\
Number of feet examined & 24 & 17 & - \\
Heel & $0.744(0.066)$ & $0.700(0.094)$ & NS \\
Midfoot & $0.158(0.07)$ & $0.207(0.089)$ & $<0.05$ \\
Lateral forefoot & $0.224(0.065)$ & $0.0269(0.068)$ & $<0.05$ \\
Toes & $0.292(0.109)$ & $0.214(0.08)$ & $<0.01$ \\
\hline \multirow{2}{*}{ Values are mean (SD). } & & &
\end{tabular}




\section{Take home message}

In patients with chronic ankle instability, there is a significant delay to the time of peak force under the central and lateral forefoot and the toes. In addition, the relative forces under the heel and toes are lower and those under the midfoot and lateral forefoot are higher than normal. This suggests that there is a lateral shift of the body weight, with a slowing down of weight transfer at the beginning and end of the stance.

\section{Authors' affiliations}

M Nyska, S Shabat, G Mann, Department of Orthopaedic Surgery, Foot and Ankle Service, Sapir Medical Center, Kfar-Saba and Tel-Aviv Sackler Medical Faculty, Israel

A Simkin, M Neeb, Y Matan, Department of Orthopaedic Surgery, Foot and Ankle Service, Hadassah Medical Center, Hebrew University, Jerusalem 91240, Israel

\section{REFERENCES}

1 Boruta PM, Bishop JO, Braly WG, et al. Acute lateral ankle ligament injuries: a literature review. Foot Ankle 1990;11:107-13.

2 Garrick JG. The frequency of injury, mechanism of injury, and epidemiology of ankle sprains. Am J Sports Med 1977;5:241-2.

3 Jackson DW, Ashley RL, Powell JW. Ankle sprains in young athletes. Relation of severity and disability. Clin Orthop 1974;101:201-15.
4 Domain WR, Staples OS, Russell SW. Residual disability following acute ankle sprains. J Bone Joint Surg [Am] 1955;37:1237-40.

5 Klenerman L. The management of sprained ankle. J Bone Joint Surg [Br] 1998;80:11-12.

6 Ashton-Miller JA, Ottaviani RA, Hutchinson C, et al. What best protects the inverted weightbearing ankle against further inversion? Am J Sports Med 1996;24:800-8.

7 Peters JW, Trevino SG, Renstrom PA. Chronic lateral ankle instability. Foot Ankle 1991;12:182-91.

8 Milgrom C, Shlamkovitch N, Finestone A, et al. Risk factors for lateral ankle sprain: a prospective study among military recruits. Foot Ankle $1991 ; 12: 26-30$.

9 Snyder RB, Lipscomb AB, Johnston RK. The relationships of tarsal coalitions to ankle sprains in athletes. Am J Sports Med $1981 ; 9: 313-17$.

10 Nef W, Gerber NJ. Hypermobility syndrome. When too much activity causes pain. Schweiz Med Wochenschr 1998;128:302-10.

11 Nyska M. Amir H, Porath A, et al. Radiological assessment of a modified anterior drawer test of the ankle. Foot Ankle 1992;13:400-3.

12 Freeman MA, Dean MR, Hanham IW. The etiology and prevention of functional instability of the foot. J Bone Joint Surg [Br] 1965;47:678-85.

13 Nyska M, Porat A, Howard CB, et al. Radiological assessment of chronic instability of the ankle. Journal of Sports Traumatology 1993;15:193-8.

14 Clanton TO. Instability of the subtalar joint. Orthop Clin North Am 1989;20:583-91.

15 Konradsen L, Olesen S, Hansen HM. Ankle sensorimotor control and eversion strength after acute ankle inversion injuries. Am J Sports Med 1998:26:72-7.

16 Lofvenberg R, Karrholm J, Sundelin G, et al. Prolonged reaction time in patients with chronic lateral instability of the ankle. Am J Sports Med 1995;23:414-17.

17 Becker HP, Rosenbaum D, Claes L, et al. Dynamic pedography for assessing functional ankle joint instability. Unfallchirurg 1997;100:133-9.

18 Louwerens JW, van Linge B, de Klerk LW, et al. Peroneus longus and tibialis anterior muscle activity in the stance phase. A quantified electromyographic study of 10 controls and 25 patients with chronic ankle instability. Acta Orthop Scand 1995;66:517-23.

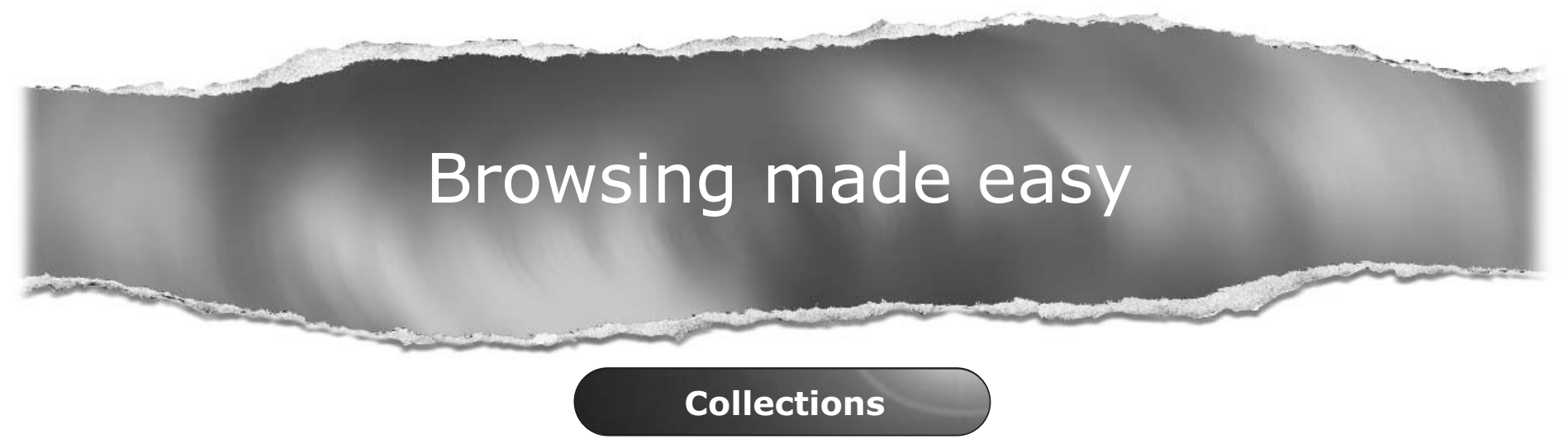

With a single click Collections allows you to find all articles that have been published in your chosen subject. Select from over 200 clinical and non-clinical topic collections and/or cross search other specialist journals, the BMJ and Cochrane Reviews 